\title{
Gradient-based stochastic estimation of the density matrix
}

\author{
Zhentao Wang, ${ }^{1}$ Gia-Wei Chern, ${ }^{2}$ Cristian D. Batista, ${ }^{1,3}$ and Kipton Barros ${ }^{4, a)}$ \\ ${ }^{1)}$ Department of Physics and Astronomy, The University of Tennessee, Knoxville, TN 37996, \\ USA \\ ${ }^{2)}$ Department of Physics, University of Virginia, Charlottesville, VA 22904, USA \\ 3) Quantum Condensed Matter Division and Shull-Wollan Center, Oak Ridge National Laboratory, Oak Ridge, \\ TN 37831, USA \\ 4) Theoretical Division and CNLS, Los Alamos National Laboratory, Los Alamos, NM 87545, \\ $U S A$
}

Fast estimation of the single-particle density matrix is key to many applications in quantum chemistry and condensed matter physics. The best numerical methods leverage the fact that the density matrix elements $f(H)_{i j}$ decay rapidly with distance $r_{i j}$ between orbitals. This decay is usually exponential. However, for the special case of metals at zero temperature, algebraic decay of the density matrix appears and poses a significant numerical challenge. We introduce a gradient-based probing method to estimate all local density matrix elements at a computational cost that scales linearly with system size. For zero-temperature metals the stochastic error scales like $S^{-(d+2) / 2 d}$, where $d$ is the dimension and $S$ is a prefactor to the computational cost. The convergence becomes exponential if the system is at finite temperature or is insulating.

\section{INTRODUCTION}

Many topics in quantum chemistry and condensed matter physics involve an effective Hamiltonian,

$$
\hat{\mathcal{H}}=\sum_{i, j=1}^{N} c_{i}^{\dagger} H_{i j} c_{j},
$$

which is quadratic in fermionic creation and annihilation operators $\left(c_{i}^{\dagger}\right.$ and $\left.c_{i}\right)$. Examples include densityfunctional tight-binding model $\$^{112}$ for molecular dynamics simulation, $\frac{3}{2}$ Kondo lattice models of itinerant electrons interacting with localized magnetic moments,, Falicov-Kimball models of metal-insulator transitions, and Bogoliubov-de Gennes equations for superconductivity [6] The index $i$ specifies a single-particle wave function (position, spin, orbital index, etc.). The electronic free energy in the grand canonical ensemble is given by $\Omega=-k_{B} T \ln Z$, where $k_{B}$ is the Boltzmann constant and $T$ is the temperature. The partition function is $Z=\operatorname{tr} e^{-\beta\left(\hat{\mathcal{H}}-\mu \hat{N}_{e}\right)}$, with $\beta=1 / k_{B} T$, chemical potential $\mu$, and electron number $\hat{N}_{e}=\sum_{i=1}^{N} c_{i}^{\dagger} c_{i}$. Evaluating the above trace over fermions yields

$$
\Omega=\sum_{\epsilon} g(\epsilon)=\operatorname{tr} g(H)
$$

where $\{\epsilon\}$ are the eigenvalues of the single-particle Hamiltonian matrix $H$, and

$$
g(x)=-\beta^{-1} \ln \left[1+e^{-\beta(x-\mu)}\right] .
$$

Note that the derivative of $g(x)$ is the usual Fermi function,

$$
\frac{\mathrm{d} g(x)}{\mathrm{d} x}=f(x)=\frac{1}{e^{\beta(x-\mu)}+1} .
$$

\footnotetext{
a) Electronic mail: kbarros@lanl.gov
}

Consequently, the derivative of the free energy gives the density matrix,

$$
f(H)=\mathrm{d} \Omega / \mathrm{d} H^{T} .
$$

Density matrix elements represent two-body correlations, $f(H)_{i j}=\left\langle c_{j}^{\dagger} c_{i}\right\rangle$. Diagonal elements $f(H)_{i i}$ give the charge localized at $i$. The expected electron number $\left\langle\hat{N}_{e}\right\rangle$ is

$$
N_{e}=\operatorname{tr} f(H)
$$

Efficient estimation of the density matrix, especially for metals, is the central topic of this paper. Our primary motivation is to enable dynamical simulations of effectively classical degrees of freedom $\left\{\mathbf{x}_{1}, \mathbf{x}_{2} \ldots\right\}$. For example, in quantum molecular dynamics, $\mathbf{x}_{\alpha}$ may be positions of nuclei evolving classically under the Born Oppenheimer approximation! $7-11$ In applications to itinerant magnets, $\mathbf{x}_{\alpha}$ may represent a field of local moments 12$]$ The $N \times N$ single-particle Hamiltonian $H$ evolves with the dynamical variables $\mathbf{x}_{\alpha}$. The electronic free energy $\Omega$ may be augmented with classical interactions solely involving the $\mathbf{x}_{\alpha}$; such interactions are straightforward to handle, and we ignore them here.

The dynamics of $\mathbf{x}_{\alpha}$ is driven by effective forces associated with energy derivatives. Referring to Eq. (5), the chain rule yields

$$
-\frac{\partial \Omega}{\partial \mathbf{x}_{\alpha}}=-\sum_{i j} f(H)_{j i} \frac{\partial H_{i j}}{\partial \mathbf{x}_{\alpha}}
$$

The matrix $\partial H / \partial \mathbf{x}_{\alpha}$, for each $\alpha$, is typically highly localized and easy to compute. At every dynamical time-step, a key numerical challenge is to calculate density matrix elements $f(H)_{i j}$ for nearby states $i$ and $j$.

Direct diagonalization of the single-particle Hamiltonian $H$ is possible but the $\mathcal{O}\left(N^{3}\right)$ cost would severely limit system sizes. Better methods take advantage of the sparsity of $H$. In a real-space basis, the elements 
$H_{i j}$ typically decay exponentially with spatial distance $r_{i j}=\left|\mathbf{r}_{i}-\mathbf{r}_{j}\right|$. If the system is either insulating or at finite temperature, then the density matrix $f(H)_{i j}$ also decays exponentially in $r_{i j} \cdot 19$ A rich set of algorithms have emerged to calculate $f(H)$ by leveraging its sparsity. 2021 Methods based upon iterated self-multiplication of sparse matrices $22-24$ enable quantum molecular dynamics simulations with up to millions of atoms. $9 \mid 10$

In the case of metals at zero temperature, however, $f(H)_{i j}$ decays just algebraically in $r_{i j}$. Consequently, state-of-the-art methods based upon sparse matrix-matrix multiplication are infeasible. Here we consider instead stochastic methods that require only sparse matrix-vector multiplication.

In a direct probing approach, one may approximate $f(H) \approx[f(H) R] R^{\dagger} \cdot{ }^{25 \mid 26}$ The random matrix $R$ contains $N \times S$ elements. The parameter $S$ becomes a prefactor to the computational cost and controls accuracy. Although never explicitly constructed, the outer product matrix $R R^{\dagger}$ is an unbiased approximation to the $N \times N$ identity matrix. References 27 and 28 introduce a coloring strategy to design $R$ to best leverage the spatial decay of $f(H)$. With this strategy, we show that the stochastic error for direct probing scales like $\Delta f \sim S^{-(d+1) / 2 d}$ for bulk $d$-dimensional metals at zero temperature.

Inspired by Eq. (5) and the favorable decay properties of $g(H)$, we introduce a gradient-based probing approximation, $f(H) \approx\left(\mathrm{d} / \mathrm{d} H^{T}\right) \operatorname{tr} g(H) R R^{\dagger}$, and show that its error scales like $\Delta f \sim S^{-(d+2) / 2 d}$. This approximation scheme and its rapid convergence are our main results.

Crucially, the accuracy in estimating density matrix elements is independent of system size $N$. Our probing method thus enables truly linear-scaling dynamical simulations of metals. Furthermore, to a first approximation, the unbiased stochastic errors in the forces [cf. Eq. (7)] can be absorbed into the noise term of a Langevin thermostat.12/29 31 Alternatively, for fixed matrix $R$, gradient-based probing yields conservative forces that generate time-reversible dynamics. Empirically, we commonly find that $S \lesssim 100$ random column vectors enable accurate dynamical simulations over a wide range of temperatures.

\section{STOCHASTIC TRACE ESTIMATION}

We begin with stochastic estimation of the matrix traces, Eqs. (6) and (2). One may approximate the electron number as

$$
N_{e}=\operatorname{tr} f(H) \approx \sum_{s=1}^{S} r^{(s) \dagger} f(H) r^{(s)}=\operatorname{tr} R^{\dagger} f(H) R,
$$

where $R$ is a stochastic, $N \times S$ matrix composed of column vectors $r^{(s)}$. Typically, $S \ll N$. The approximation error is 27

$$
\Delta N_{e}=\operatorname{tr} f(H)\left(R R^{\dagger}-I\right) .
$$

Observe that the approximation is unbiased, $\left\langle\Delta N_{e}\right\rangle=0$, provided that, on average, $\left\langle R R^{\dagger}\right\rangle=I$. The quality of the approximation will typically improve with the number of column vectors $S$.

The free energy may be approximated similarly,

$$
\Omega \approx \operatorname{tr} R^{\dagger} g(H) R .
$$

The free energy error analysis is completely analogous to that of $\Delta N_{e}$, which we will present below.

A remark on our numerical implementation: The matrix-vector products $f(H) r^{(s)}$ and $g(H) r^{(s)}$ can be approximated at a cost that scales linearly with system size $N$. In our approach we expand $f(H)$ and $g(H)$ in Chebyshev polynomials over $H$ using the Kernel Polynomial Method (Appendix A), 32,34 this method is simple and amenable to a gradient transformation (Appendix B [12]35 Other methods, such as rational approximation, $36 \mid 37$ are also possible, at least in principle. $\mathrm{Al}-$ ternative trace estimators have also been proposed $\underline{38 \mid 39}$

By numerically inverting the approximation of Eq. (8), we can allow the chemical potential $\mu$ to vary according to a fixed electron number $N_{e}$. Within this canonical ensemble, the density matrix $f(H)$ may still be interpreted as the gradient of the characteristic free energy. Appendix D presents the details of this transformation.

\section{A. Uncorrelated probing}

One possible choice for the stochastic matrix $R$ is

$$
R_{i s}=\zeta_{i s} / \sqrt{S}
$$

where $\zeta_{i s}$ are uncorrelated random numbers that satisfy $\left\langle\zeta_{i s} \zeta_{j t}^{*}\right\rangle=\delta_{i j} \delta_{s t}$. It is advantageous to constrain $\left|\zeta_{i s}\right|=1$ such that $\left(R R^{\dagger}\right)_{i i}=1$ and only off-diagonal elements contribute to the error $\Delta N_{e}$. If we select $\zeta_{i s}$ to be complex numbers with uniformly random phases ${ }^{40}$ then one can show that the variance of the stochastic error is

$$
\operatorname{var}\left[\Delta N_{e}\right]=\frac{1}{S} \sum_{i<j}\left|f(H)_{i j}\right|^{2} \quad(R \text { uncorrelated })
$$

Observe that approximation (8) implicitly benefits from the smallness of the off-diagonal elements of $f(H)$.

The idea to estimate traces using uncorrelated random column vectors appeared in Refs. 41 and 42 and has been employed by the Kernel Polynomial Method! 32 Early related methods include Refs. 43,45. Since then, similar techniques have found practical applications in quantum chemistry and electronic structure. 66 - 51 As we discuss below, it is often preferable to design $R$ as a whole, rather than work with its column vectors $r^{(s)}$ independently.

\section{B. Optimized probing}

Approximation (8) can be improved by optimizing $R$ to take better advantage of the spatial decay properties 


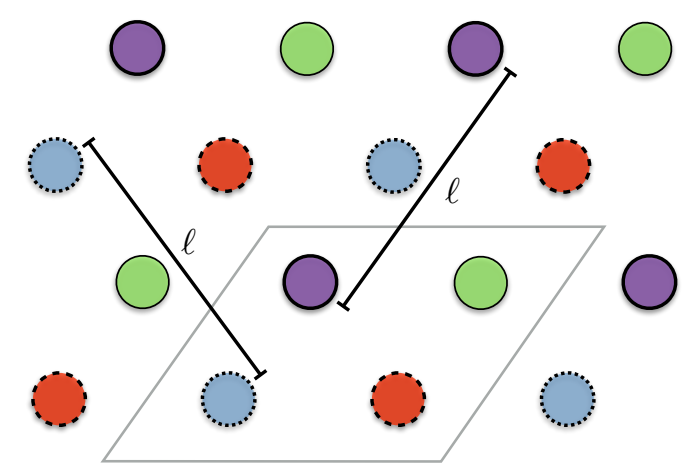

Figure 1. Coloring sites on the triangular lattice. With $S$ colors (here 4), pairs of same-color sites are separated by at least $\ell=S^{1 / 2}$ lattice constants (here 2). In the general $d$ dimensional case we expect to find a coloring such that samecolor sites are separated by a distance $\ell$ that scales like $S^{1 / d}$.

of $f(H)_{i j}$. The idea, presented in Refs. 27 and 28 , is to construct $R$ such that the elements $\left(R R^{\dagger}\right)_{i j}$ are zero whenever $f(H)_{j i}$ is large, thus eliminating the largest contributions to the error in Eq. (9). Here, we make use of the physical property, to be discussed below, that $f(H)_{i j}$ decays with spatial distance $r_{i j}$ between orbitals $i$ and $j$.

The first step in designing the $N \times S$ matrix $R$ is to assign a color $c(i) \in\{1,2, \ldots S\}$ to each localized orbital $i$. We employ the heuristic that different colors should be assigned to sites $i$ and $j$ whose separation $r_{i j}$ is small. That is, given $S$ colors, we seek a coloring that satisfies

$$
c(i) \neq c(j) \quad \text { if } \quad r_{i j}<\ell,
$$

with the largest possible distance $\ell$. If the sites are distributed roughly uniformly in $d$-dimensional space, we expect to find a coloring that satisfies

$$
\ell \sim S^{1 / d}
$$

An optimal strategy for coloring the triangular lattice is illustrated in Fig. 1; observe that with $S$ colors, we can separate same-color sites by $\ell=\sqrt{S}$ lattice constants. The case of a one-dimensional lattice is even simpler: the optimal coloring, $c(i)=1+(i-1) \bmod S$, separates same-color sites by $\ell=S$ lattice constants.

Given a coloring, we can replace the uncorrelated matrix of Eq. (11) with the optimized one

$$
R_{i s}=\delta_{c(i), s} \zeta_{i},
$$

where $\zeta_{i}$ are uncorrelated random numbers. The outer product matrix becomes

$$
\left(R R^{\dagger}\right)_{i j}=\delta_{c(i), c(j)} \zeta_{i} \zeta_{j}^{*}
$$

As before, we constrain $\left|\zeta_{i}\right|=1$ such that $\left(R R^{\dagger}\right)_{i i}=$ 1. The off-diagonal elements $\left(R R^{\dagger}\right)_{i j}$ are mostly zero, except for orbital pairs $(i, j)$ that share the same color,
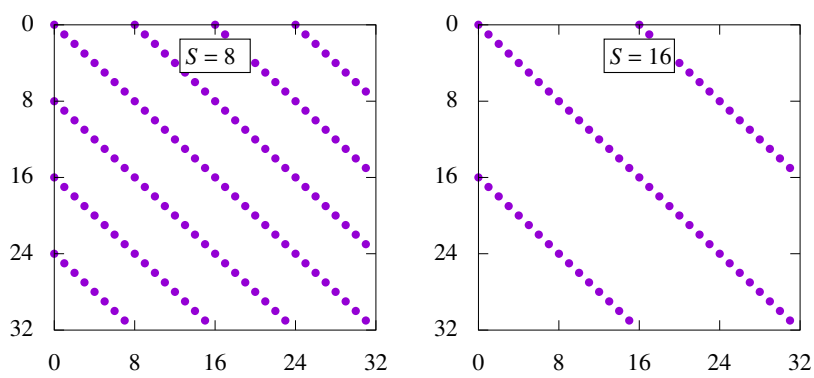

Figure 2. Structure of the matrix $R R^{\dagger}$ for an optimal coloring on the one-dimensional lattice. The diagonal elements are exactly one. The closest non-zero elements are a distance $S$ away from the diagonal elements. This matrix structure enhances the convergence of trace estimates $\operatorname{tr} A \approx \operatorname{tr} R^{\dagger} A R$ as a function of the number of colors $S$.

$c(i)=c(j)$. Figure 2 illustrates the sparsity structure of $R R^{\dagger}$ in the one-dimensional case.

As before, the stochastic error is given by Eq. (9). Its variance can be calculated by inserting Eq. (16). After some analysis, we obtain a sum over same-color, offdiagonal elements,

$$
\operatorname{var}\left[\Delta N_{e}\right]=\sum_{i<j} \delta_{c(i), c(j)}\left|f(H)_{i j}\right|^{2} \quad(R \text { optimized }) .
$$

Compared to the uncorrelated result, Eq. (12), we lose a prefactor of $1 / S$ but gain the constraint $c(i)=c(j)$, which eliminates all but $\sim 1 / S$ of the terms. The great advantage of optimized probing is that the remaining terms correspond to orbital pairs $(i, j)$ that satisfy $r_{i j} \geq \ell$, for which $f(H)_{i j}$ is small. To quantify the numerical advantage of probing, we must first determine the actual smallness of relevant matrix elements $f(H)_{i j}$ and $g(H)_{i j}$.

Finally, we note that in the limit $S \rightarrow N$, each orbital gets a unique color, $c(i)=i$, and the stochastic error in Eq. (17) disappears. Our theoretical analysis will focus on the regime $1 \ll S \ll N$.

\section{Spatial decay of density and energy matrices}

We focus our analysis on metallic systems at zero temperature, for which the density matrix $f(H)$ decays most slowly, thus posing the greatest numerical challenge. For simplicity, here we assume a single electronic band with quadratic dispersion $\epsilon_{\mathbf{k}}=k^{2} / 2$, partially filled up to a chemical potential $\mu=k_{F}^{2} / 2$. We work in arbitrary spatial dimension $d$. At zero temperature the Fermi function reduces to the Heaviside function, $f\left(\epsilon_{\mathbf{k}}\right)=\Theta\left(\mu-\epsilon_{\mathbf{k}}\right)$ and the real-space density matrix elements can be calculated 

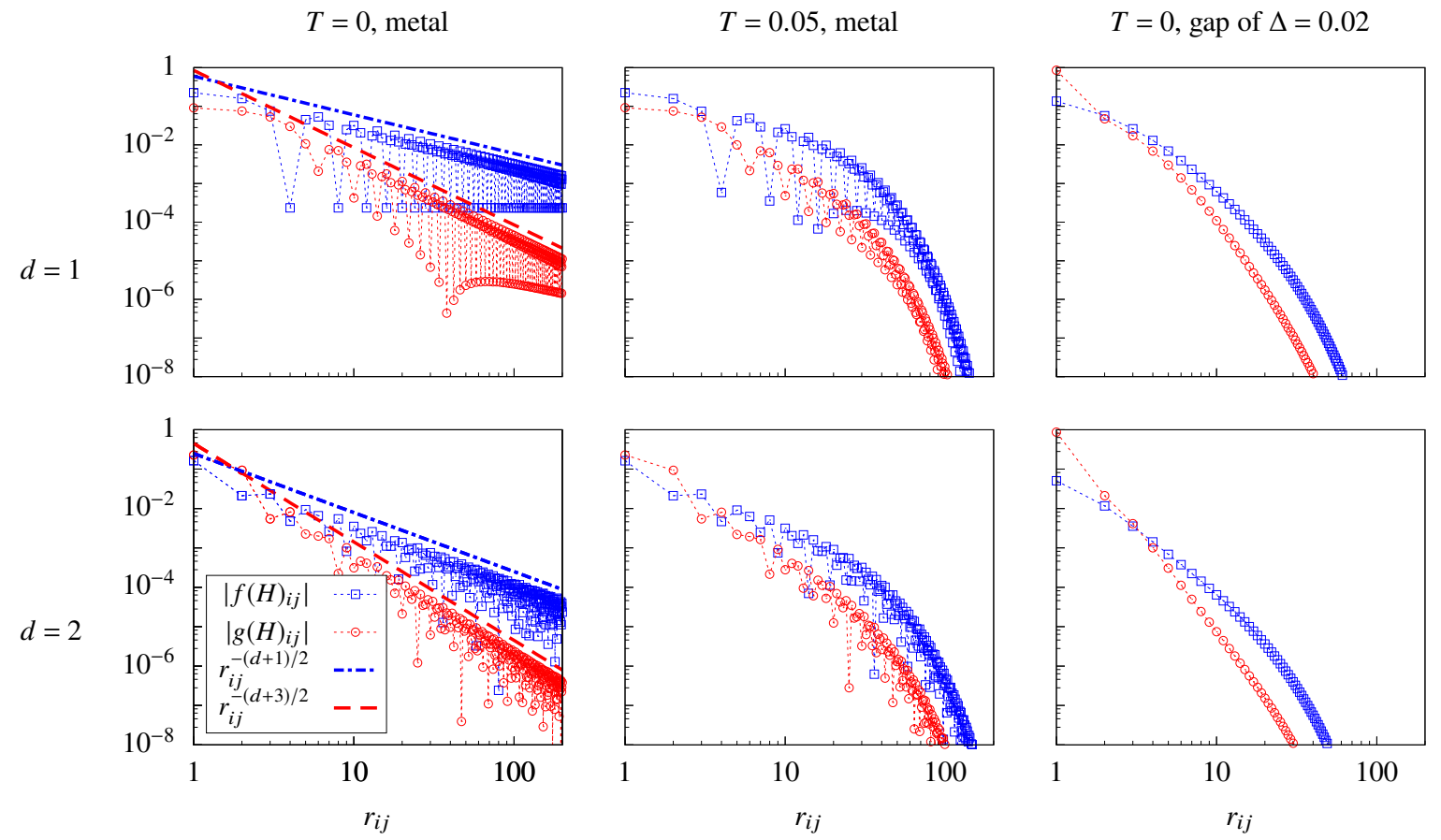

Figure 3. Decay of the density and energy matrices, $f(H)_{i j}$ and $g(H)_{i j}$, as a function of distance $r_{i j}$ between localized orbitals $i$ and $j$. For zero-temperature metals (left column), we observe power law scaling, $\left|f(H)_{i j}\right| \sim r_{i j}^{-(d+1) / 2}$ and $\left|g(H)_{i j}\right| \sim r_{i j}^{-(d+3) / 2}$, where $d$ is the spatial dimension. If there is a finite temperature $T$ (middle column), or band gap $\Delta$ (right column), the decay becomes much faster. $T$ and $\Delta$ are measured in units of the hopping constant for this model tight-binding system.

by Fourier transform $52 \mid 53$

$$
\begin{aligned}
f(H)_{i j} & =(2 \pi)^{-d} \int \mathrm{d}^{d} \mathbf{k} f\left(\epsilon_{\mathbf{k}}\right) e^{-i \mathbf{k} \cdot \mathbf{r}_{i j}} \\
& =\left(\frac{k_{F}}{2 \pi r_{i j}}\right)^{d / 2} J_{d / 2}\left(k_{F} r_{i j}\right) .
\end{aligned}
$$

We have assumed that the volume of the primitive cell is one.

For large argument $k_{F} r_{i j}$, the Bessel function of the first kind scales asymptotically as

$$
J_{d / 2}\left(k_{F} r_{i j}\right) \approx \sqrt{\frac{2}{\pi k_{F} r_{i j}}} \cos \left[k_{F} r_{i j}-\frac{\pi(d+1)}{4}\right] .
$$

Ignoring oscillations, we conclude that the density matrix elements decay as

$$
\left|f(H)_{i j}\right| \sim f_{\mathrm{dec}}\left(r_{i j}\right)=r_{i j}^{-(d+1) / 2} .
$$

We also consider the energy matrix, $g(H)$, defined via Eq. (3). At zero temperature, $g\left(\epsilon_{\mathbf{k}}\right)=\left(\epsilon_{\mathbf{k}}-\mu\right) f\left(\epsilon_{\mathbf{k}}\right)$ and the energy matrix elements become

$$
\begin{aligned}
g(H)_{i j} & =(2 \pi)^{-d} \int \mathrm{d}^{d} \mathbf{k} g\left(\epsilon_{\mathbf{k}}\right) e^{-i \mathbf{k} \cdot \mathbf{r}_{i j}} \\
& =-\frac{k_{F}}{r_{i j}}\left(\frac{k_{F}}{2 \pi r_{i j}}\right)^{d / 2} J_{d / 2+1}\left(k_{F} r_{i j}\right) .
\end{aligned}
$$

At large distances, $g(H)_{i j}$ decays as

$$
\left|g(H)_{i j}\right| \sim g_{\mathrm{dec}}\left(r_{i j}\right)=r_{i j}^{-(d+3) / 2} .
$$

We conclude that the energy matrix decays one power faster than the density matrix, Eq. (20).

At small nonzero temperature, $\bar{T}>0$, the decay of both $f_{\mathrm{dec}}\left(r_{i j}\right)$ and $g_{\mathrm{dec}}\left(r_{i j}\right)$ becomes exponential, $\left.\exp \left(-c r_{i j} T\right)\right)$, for some constant $c{ }^{54}$ Similarly, if the chemical potential lies within a small band gap of width $\Delta$, the decay also becomes exponential, $\exp \left(-c r_{i j} \Delta\right) ! \underline{55}$ Once exponential decay is introduced, a new power law prefactor may appear. For example, the density matrix for insulators with small gap may actually scale as $\left|f(H)_{i j}\right| \sim r_{i j}^{-d / 2} \exp \left(-c r_{i j} \Delta\right) ! 56157$ These exponential decays are asymptotic upper bounds. For example, a faster decay $\exp \left(-c r_{i j} \sqrt{\Delta}\right)$ has been observed along nondiagonal directions of a model insulator on the square lattice. ${ }^{58}$ Many of the above scaling bounds have been demonstrated with mathematical rigor. 59

Although Eqs. (20) and (22) were derived in the context of a model isotropic material, the power law exponents $(d+1) / 2$ and $(d+3) / 2$ are universal to bulk metals at zero temperature. We demonstrate this numerically in the context of a simple tight-binding model, $\mathcal{H}=t \sum_{\langle i j\rangle} c_{i}^{\dagger} c_{j}$, with hoppings between nearestneighbor sites, $\langle i j\rangle$. We use dimensionless units for energy $(t=1)$, temperature $\left(k_{B}=1\right)$, and length (lattice 
constant $a=1$ ). We study linear and square lattices, $d=\{1,2\}$, with $N=\left\{10^{4}, 2000^{2}\right\}$ lattice sites, respectively. For metals, we fix the electron number to quarter filling fraction. To realize an insulating gap of width $\Delta$, we switch our model system to half filling and introduce a uniform magnetic field to split the spin-up and -down bands.

We use the Kernel Polynomial Method to expand $f(H)$ and $g(H)$, as described in Appendix A To calculate the matrix elements with high precision, we do not apply any stochastic approximation, and we use an extremely large polynomial order, $M=10^{5}$.

Figure 3 shows the matrix decays for this model system. At zero temperature, we observe the expected power laws of $f_{\mathrm{dec}}\left(r_{i j}\right)$ and $g_{\mathrm{dec}}\left(r_{i j}\right)$. Scatter is associated with the oscillatory nature of Bessel functions, Eq. (19). Introducing either finite temperature $(T=0.05)$ or gap $(\Delta=0.02)$ leads to much faster matrix decay.

\section{Error analysis}

Armed with the decay properties of $f(H)$ and $g(H)$, we can now quantify the stochastic errors $\Delta N_{e}$ and $\Delta \Omega$ for probing estimates of the electron number, $N_{e}=\operatorname{tr} f(H)$, and grand canonical free energy, $\Omega=\operatorname{tr} g(H)$, respectively.

If the matrix $R$ is constructed as a concatenation of $S$ uncorrelated random column vectors, then Eq. 12 gives the variance of these errors as a double sum over orbitals. The first sum, over $i$, is unconstrained, yielding a factor of system size $N$. The second sum, over $j$, only contributes when orbitals $i$ and $j$ are local, due to the sufficiently fast spatial decay of $f(H)$ and $g(H)$. We conclude

$$
\operatorname{var}\left[\Delta N_{e}\right] \sim \operatorname{var}[\Delta \Omega] \sim N \times S^{-1} \quad(R \text { uncorrelated }) .
$$

The standard deviations of $\Delta N_{e}$ and $\Delta \Omega$ thus scale like $\sqrt{N / S}$. Consequently, probing estimates of intensive quantities such as $N_{e} / N$ and $\Omega / N$ actually improve with increasing system size, which can be attributed to selfaveraging. $[32$

We see a significant improvement when using the optimized matrix $R$ of Eq. (15) with well selected colors $c(i)=\{1,2, \ldots S\}$. For metallic systems at zero temperature and spatial dimension $d$, the variance of Eq. (17) becomes

$$
\begin{aligned}
\operatorname{var}\left[\Delta N_{e}\right] & \sim N \times S^{-(d+1) / d}, \\
\operatorname{var}[\Delta \Omega] & \sim N \times S^{-(d+3) / d} \quad(R \text { optimized }) .
\end{aligned}
$$

The factor $N$ again appears due to a single unconstrained sum over orbitals. The dependence on $S$ follows from the fact that same-color sites are separated by a distance of at least $\ell \sim S^{1 / d}$ [cf. Eqs. (13) and (14)]. Consequently, the largest terms in Eq. (17) are of order $f_{\operatorname{dec}}(\ell)^{2}$ and $g_{\mathrm{dec}}(\ell)^{2}$, whose scaling behaviors are given by Eqs. 20 )
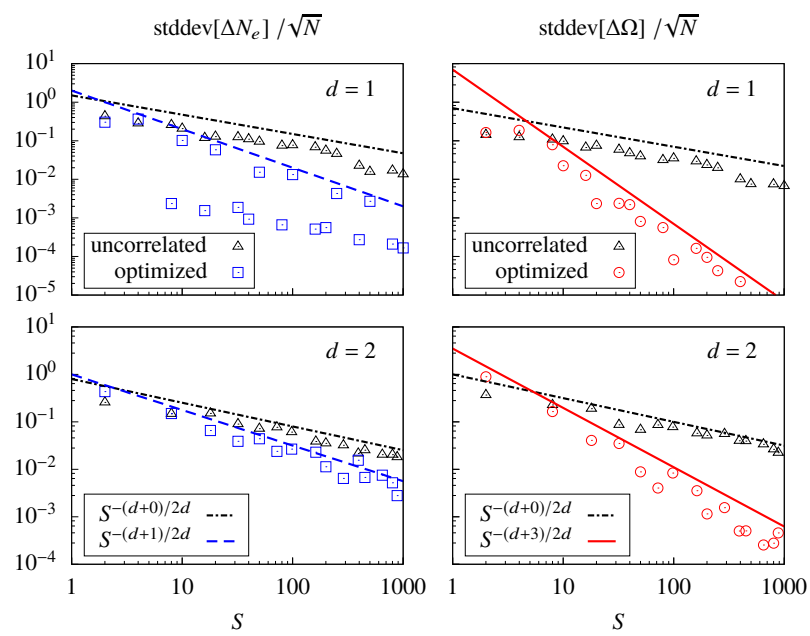

Figure 4. Stochastic errors for probing estimates of the electron number $N_{e}$ and free energy $\Omega$. With $S$ uncorrelated random vectors, Eq. 11, the errors scale as $S^{-1 / 2}$. Optimized probing, Eq. (15), yields much smaller errors. For a zero-temperature metallic system, $\Delta N_{e} \sim S^{-(d+1) / 2 d}$ and $\Delta \Omega \sim S^{-(d+3) / 2 d}$.

and 22 . We note that $f(H)$ and $g(H)$ decay sufficiently fast such that matrix elements $(i, j)$ with separation $r_{i j} \gg \ell$ do not contribute significantly to the above variances.

Figure 4 shows numerical estimation of the probing errors for zero-temperature metals. Again, we use a nearest neighbor tight-binding model in $d=\{1,2\}$ dimensions, and quarter electron filling. The lattice sizes are $N=\left\{10^{4}, 1260^{2}\right\}$ respectively. We perform the numerics with Chebyshev polynomial order $M=3000$. To estimate the standard deviations of probing errors, we repeat each probing calculation 10 times using independent samples of the random matrix $R$. Due to translation invariance of the model, we can extract an independent estimate of the stochastic errors at each lattice site, over which we average. The resulting estimates of $\operatorname{stddev}\left[\Delta N_{e}\right]$ and $\operatorname{stddev}[\Delta \Omega]$ verify the power law scaling predicted by Eqs. (24) and 25). The scatter, as a function of $S$, is not a sampling artifact; it arises due to the oscillatory decay of $f(H)_{i j}$ and $g(H)_{i j}$.

\section{DENSITY MATRIX ESTIMATION}

\section{A. Direct probing}

The trace approximation of Eq. (8) generalizes to an approximation for individual density matrix elements, $\underline{60}$

$$
f(H) \approx f(H) R R^{\dagger} .
$$

Taking the trace of both sides recovers Eq. (8) exactly. In a numerical implementation, we do not construct the full matrix $R R^{\dagger}$ explicitly. Instead, we first build $f(H) R$ and 
then use it to calculate only the desired elements $f(H)_{i j}$. Details are discussed below in Sec. IIIC.

The stochastic error of the direct approximation is

$$
\Delta f(H)^{\text {direct }}=f(H)\left(R R^{\dagger}-I\right) .
$$

Repeating the analysis of the previous section, we find that its variance scales as

$$
\operatorname{var}\left[\Delta f(H)_{i j}^{\text {direct }}\right] \sim \begin{cases}S^{-1} & (R \text { uncorrelated }) \\ S^{-(d+1) / d} & (R \text { optimized })\end{cases}
$$

for the $R$ matrices specified in Eqs. (11) and (15), respectively. To see that these results are consistent with Eqs. (23) and (24), we first observe that $N_{e}=\sum_{i} f(H)_{i i}$. Consequently, $\operatorname{var}\left[\Delta N_{e}\right]$ decomposes into a sum over $N$ contributions $\sum_{i} \operatorname{var}\left[\Delta f(H)_{i i}^{\text {direct }}\right]$, and thus scales like $N \times \operatorname{var}\left[\Delta f(H)_{i j}^{\text {direct }}\right]$. An overall factor of system size $N$ thus appears in estimates of extensive quantities such as $N_{e}$ and $\Omega$, but not local quantities such as $f(H)_{i j}$. Note that $\operatorname{var}\left[\Delta f(H)_{i j}^{\text {direct }}\right]$ is roughly independent of the choice of orbitals $i$ and $j$ provided that their distance $r_{i j}$ is small, which we will assume.

\section{B. Gradient-based probing}

A key observation in this paper is that it is possible to achieve faster stochastic convergence than with direct probing. Inserting Eq. (5) into (2), and applying approximation 10 , we find,

$$
f(H)=\frac{\mathrm{d}}{\mathrm{d} H^{T}} \operatorname{tr} g(H) \approx \frac{\mathrm{d}}{\mathrm{d} H^{T}} \operatorname{tr} g(H) R R^{\dagger},
$$

with error

$$
\Delta f(H)^{\operatorname{grad}}=\frac{\mathrm{d}}{\mathrm{d} H^{T}} \operatorname{tr} g(H)\left(R R^{\dagger}-I\right) .
$$

Interestingly, the stochastic errors of direct $(26)$ and gradient-based 29) approximation schemes are not the same,

$$
\Delta f(H)^{\text {direct }} \neq \Delta f(H)^{\mathrm{grad}} .
$$

To demonstrate the inequality in a simple context, consider substitutions $f \mapsto n H^{n-1}$ and $g \mapsto H^{n}$ for integer $n$. Then

$$
n H^{n-1}=\frac{\mathrm{d}}{\mathrm{d} H^{T}} \operatorname{tr} H^{n}
$$

but

$$
\begin{aligned}
& n H^{n-1} R R^{\dagger} \neq \\
& \quad \sum_{m=1}^{n} H^{m-1} R R^{\dagger} H^{n-m}=\frac{\mathrm{d}}{\mathrm{d} H^{T}} \operatorname{tr} H^{n} R R^{\dagger} .
\end{aligned}
$$
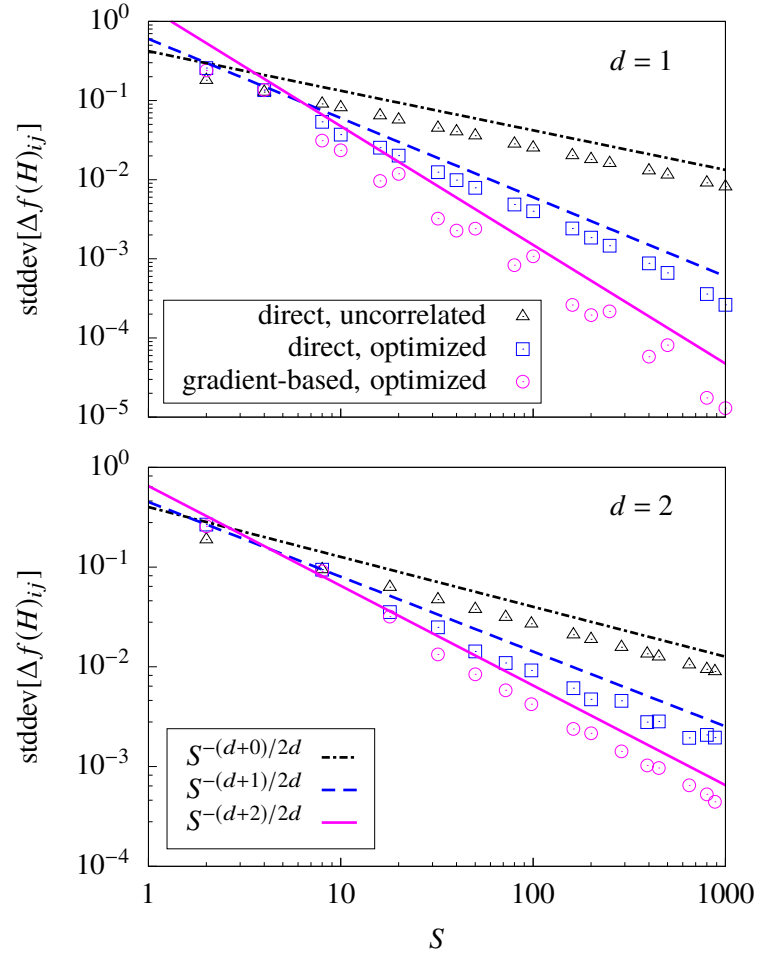

Figure 5. Stochastic errors for probing estimates of local density matrix elements $f(H)_{i j}$. We consider a zero-temperature metallic system. Using direct probing, $f(H) \approx f(H) R R^{\dagger}$, with $S$ uncorrelated random vectors, Eq. [11, the stochastic error scales like $\Delta f \sim S^{-1 / 2}$. When $R$ is optimized according to Eq. 15 , the error scales like, $\Delta f \sim S^{-(d+1) / 2 d}$. Our new gradient-based approximation, $f(H) \approx\left(\partial / \partial H^{T}\right) \operatorname{tr} g(H) R R^{\dagger}$, achieves the smallest error: $\Delta f \sim S^{-(d+2) / 2 d}$.

Inequality stems from the fact that $H$ and $R R^{\dagger}$ do not commute. Observe that the gradient-based approximation (i.e., the right hand side) may benefit from cancellations between $n$ different approximations, each unbiased. The density matrix $f(H)$ may be expanded in powers of $H$, suggesting that $\Delta f(H)^{\text {grad }}$ may similarly be smaller than $\Delta f(H)^{\text {direct }}$. After careful analysis (Appendix E) we find,

$$
\operatorname{var}\left[\Delta f(H)_{i j}^{\operatorname{grad}}\right] \sim S^{-(d+2) / d} \quad(R \text { optimized }),
$$

which is indeed superior to the direct probing approach, Eq. 28. Intuitively, we associate the smaller error of gradient-based probing with the faster decay of the energy matrix, relative to the density matrix. Surprisingly, the exponent $(d+2) / d$ is new, and halfway between the exponents associated with the decay of $f(H)$ and $g(H)$ [cf. Eqs. 24) and [25]].

\section{Numerics}

Figure 5 illustrates the accuracy of various approximation schemes for estimating $f(H)_{i j}$. We use the same 
tight-binding model and methods as in Fig. 4 Here, however, we measure the standard deviation of error $\Delta f(H)_{i j}$ for probing estimates of individual matrix elements $f(H)_{i j}$. We take $i$ and $j$ to be nearest-neighbor lattice sites $\langle i j\rangle$, but the same asymptotic scaling holds for any local matrix element (next nearest-neighbors, etc.). We confirm the power laws predicted in Eqs. 28 and (33) for zero-temperature metals.

The details of our numerical implementation are as follows. For direct probing, Eq. (26), we use the methods presented in Appendix A. We start with the Chebyshev polynomial expansion $f(H) \approx f_{M}(H)=$ $\sum_{m=0}^{M-1} c_{m}^{(f)} T_{m}(H)$ of Eq. A9. Next, we evaluate the matrix product $f_{M}(H) R$ as a linear combination of matrices $\alpha_{m}=T_{m}(H) R$, which are calculated recursively using Eq. A13. Finally, we take the outer product, $f(H)_{i j} \approx \sum_{s=1}^{S}\left[f_{M}(H) R\right]_{i s} R_{j s}^{*}$, for desired elements $(i, j)$. In typical applications, we require $f(H)_{i j}$ only if $H_{i j}$ is non-vanishing, i.e., if the distance $r_{i j}$ between orbitals $(i, j)$ is very small. The total computational cost to approximate $f(H)$ thus scales like $\mathcal{O}(N M S)$.

The gradient-based probing approximation of Eq. (29) is more subtle to implement. We begin with the free energy approximation described in Appendix A. Specifically, we use the recursive procedure defined by Eqs. A12-A14 to calculate $\Omega \approx \tilde{\Omega}=\operatorname{tr} R^{\dagger} f_{M}(H) R$. Taking the exact derivative of the approximate free energy yields the desired density matrix approximation, $f(H) \approx \mathrm{d} \tilde{\Omega} / \mathrm{d} H^{T}$. Appendix $\mathrm{B}$ describes the procedure to calculate matrix elements $\mathrm{d} \Omega / \mathrm{d} H_{j i}$ using reverse-mode automatic differentiation. Crucially, we calculate all relevant matrix elements simultaneously, such that the procedures to estimate $\Omega$ and $f(H)$ both scale like $\mathcal{O}(N M S)$.

We save a factor of 2 in the computational cost by using a product identity for Chebyshev polynomials, as described in Appendix C.

\section{CONCLUSIONS}

Our aim is efficient estimation of the density matrix $f(H)$, where $H$ is the single-particle Hamiltonian. The greatest numerical challenge appears for metals at zero temperature; in this case, $f(H)_{i j}$ decays like $r_{i j}^{-(d+1) / 2}$, where $r_{i j}$ is the distance between orbitals and $d$ the spatial dimension.

In a direct probing approach, one may approximate

$$
f(H) \approx f(H) R R^{\dagger}
$$

where $R$ is a suitable $N \times S$ matrix. If the elements of $R$ are random and uncorrelated, the stochastic error associated with estimates of local density matrix elements scales as $\Delta f \sim S^{-1 / 2}$. Better approaches take advantage of the spatial decay of $f(H)$. Optimized probing carefully assigns a color $c(i) \in\{1,2, \ldots S\}$ to each local orbital $i$, such that nearby orbitals have different colors. 28
Then the $R$ matrix of Eq. 15 yields improved approximations, with error $\Delta f \sim S^{-(d+1) / 2 d}$ for metals at zero temperature.

In this paper, we introduce a new gradient-based probing technique,

$$
f(H) \approx \frac{\mathrm{d}}{\mathrm{d} H^{T}} \operatorname{tr} g(H) R R^{\dagger},
$$

where $\mathrm{d} g(x) / \mathrm{d} x=f(x)$. This approximation would become exact if we were to replace $R R^{\dagger}$ with the identity. We show that the energy matrix elements $g(H)_{i j}$ decay like $r_{i j}^{-(d+3) / 2}$ for metals at zero temperature. Equation 35 with optimized $R$ leverages this faster matrix decay; careful analysis shows that the stochastic error scales like $\Delta f \sim S^{-(d+2) / 2 d}$, which we have confirmed numerically.

By applying reverse-mode automatic differentiation to the Kernel Polynomial Method, we demonstrate an efficient implementation strategy for gradient-based probing. The computational cost to estimate $\mathcal{O}(N)$ local elements $f(H)_{i j}$ scales like $\mathcal{O}(N M S)$ where $M$ is the polynomial expansion order.

Previous linear-scaling methods have largely focused on systems for which the density matrix decays exponentially with distance. In such cases, gradient-based probing also converges exponentially quickly. Quantitative comparison with previous state-of-the-art implementations $\$ 9|10| 61$ will require experimentation, and is a topic for future work. A clear advantage of gradientbased probing, however, is that it continues to provide a high-quality, linear-scaling, and unbiased approximation to density matrix elements for metals at very low temperatures. Our GPU-optimized implementation has enabled simulations of magnetic moment dynamics on lattices of unprecedented size! ${ }^{12}[18$ Gradient-based probing can potentially also be applied to realistic quantum chemistry models, e.g. Kohn-Sham density functional theory along the lines of Refs. 47 and 51 .

\section{SUPPLEMENTARY MATERIAL}

See supplementary material for a stand-alone, minimal Python code that demonstrates gradient-based probing.

\section{ACKNOWLEDGMENTS}

We thank A. M. N. Niklasson, A. F. Voter, H. Suwa, and the anonymous referees for their encouragement and helpful suggestions. Work performed at LANL was supported by the Laboratory Directed Research and Development (LDRD) program. C. D. B. and G.-W. C. were supported by the Center for Materials Theory as a part of the Computational Materials Science (CMS) program, funded by the U.S. Department of Energy, Office of Science, Basic Energy Sciences, Materials Sciences and Engineering Division. 


\section{Appendix A: Kernel Polynomial Method}

\section{Expansion of the density of states}

The density of states,

$$
\rho(x)=\sum_{\epsilon} \delta(x-\epsilon),
$$

is a representation of the eigenvalues $\epsilon$ of the Hamiltonian $H$. The Kernel Polynomial Method 32 approximates

$$
\rho(x) \approx \rho_{M}(x)=\sum_{m=0}^{M-1} g_{m}^{M} \frac{w(x)}{q_{m}} \mu_{m} T_{m}(x)
$$

using Chebyshev polynomials $T_{m}(x)=\cos (m \arccos x)$ up to order $M$, and is valid in the range $|x| \leq 1$. The moments

$$
\mu_{m}=\int_{-1}^{+1} \rho(x) T_{m}(x) \mathrm{d} x=\operatorname{tr} T_{m}(H)
$$

are essentially the Fourier transform of $\rho(x)$ in the variable $\theta=\arccos x$. The trace representation is valid assuming that the eigenvalues of $H$ satisfy $|\epsilon|<1$. Given an unscaled Hamiltonian $H_{0}$, we use the Lanczos method to estimate its extreme eigenvalues $\epsilon_{\min }$ and $\epsilon_{\max },{ }^{62}$ from which we define $H=2\left(H_{0}-\epsilon_{\min }\right) /\left(\epsilon_{\max }-\epsilon_{\min }\right)-I$.

Equation A2 with $M \rightarrow \infty$ and $g_{m}^{M} \rightarrow 1$ follows from completeness of the Chebyshev polynomials and orthogonality under the weights

$$
\begin{aligned}
w(x) & =\left(1-x^{2}\right)^{-1 / 2}, \\
q_{m} & =\left\{\begin{array}{ll}
\pi & m=0 \\
\pi / 2 & m \geq 1
\end{array} .\right.
\end{aligned}
$$

Simple truncation, $g_{m}^{M}=1$, at finite $M$ would lead to Gibbs oscillations. To damp these oscillations, we instead select coefficients

$$
g_{m}^{M}=\frac{(M-m+1) \cos \frac{\pi m}{M+1}+\sin \frac{\pi m}{M+1} \cot \frac{\pi}{M+1}}{M+1}
$$

corresponding to the Jackson kernel 34 63/64 With this choice, $\rho_{M}(x)$ is a strictly non-negative approximation to $\rho(x)$, and converges uniformly in the limit $M \rightarrow \infty$. Figure 6 illustrates $\rho_{M}(x)$ for various $M$.

\section{Expansion of matrix functions}

The approximate density of states $\rho_{M}(x)$ enables trace estimates,

$$
\begin{aligned}
\operatorname{tr} \phi(H)= & \int_{-1}^{+1} \rho(x) \phi(x) \mathrm{d} x \\
& \approx \int_{-1}^{+1} \rho_{M}(x) \phi(x) \mathrm{d} x=\sum_{m=0}^{M-1} c_{m}^{(\phi)} \mu_{m}
\end{aligned}
$$
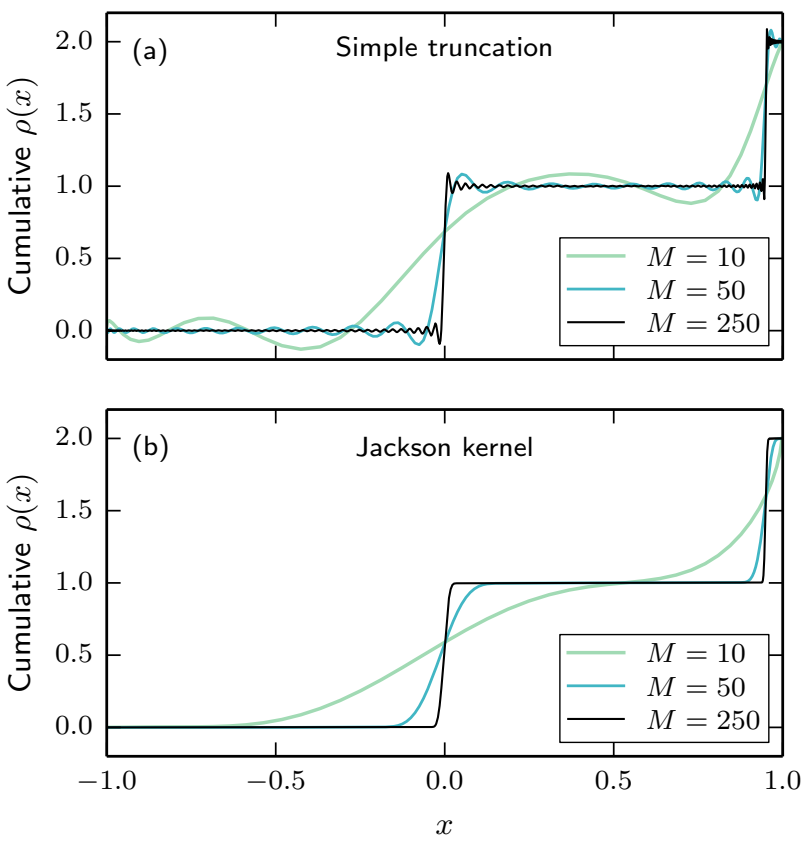

Figure 6. Kernel polynomial approximation, Eq. A2, to the density of states $\rho_{M}(x) \approx \rho(x)=\delta(x)+\delta(x-0.9)$. To aid visualization, we plot $\int_{-1}^{x} \rho_{M}\left(x^{\prime}\right) \mathrm{d} x^{\prime}$. (a) Simple truncation of the Chebyshev expansion $\left(g_{m}^{M}=1\right)$ leads to Gibbs oscillations. (b) The coefficients A6 associated with the Jackson kernel optimally damp such artifacts. Note that energy resolution is best when $x \rightarrow \pm 1$.

for any matrix function $\phi(H)$. Estimates of the free energy, $\Omega=\operatorname{tr} g(H)$, and electron number, $N_{e}=\operatorname{tr} f(H)$, follow directly. The coefficients

$$
c_{m}^{(\phi)}=\int_{-1}^{+1} g_{m}^{M} \frac{w(x)}{q_{m}} \phi(x) T_{m}(x) \mathrm{d} x
$$

may be interpreted as a Chebyshev polynomial expansion of $\phi(H)$,

$$
\phi(H) \approx \phi_{M}(H)=\sum_{m=0}^{M-1} c_{m}^{(\phi)} T_{m}(H)
$$

Direct evaluation of $\operatorname{tr} \phi_{M}(H)$ using Eq. A3 reproduces the same approximation as in Eq. (A7).

The definite integrals of Eq. A8 require care to evaluate. Chebyshev-Gauss quadrature ${ }^{65}$ gracefully handles the $x= \pm 1$ singularities of the weight function $w(x)=1 / \sqrt{1-x^{2}}$. The result is

$$
c_{m}^{(\phi)} \approx \frac{\pi g_{m}^{M}}{N_{M} q_{m}} \sum_{n=0}^{N_{M}-1} \cos \left(m \theta_{n}\right) \phi\left(\cos \theta_{n}\right),
$$

with $\theta_{n}=\pi\left(n+\frac{1}{2}\right) / N_{M}$. A reasonable choice for the number of quadrature points is $N_{M}=2 M$, where $M$ is the polynomial expansion order ${ }^{34}$ The fast discrete cosine transform of the second kind (DCT-II) can be used to calculate all $c_{m}^{(\phi)}$ at $\operatorname{cost} \mathcal{O}\left(N_{M} \ln N_{M}\right)$. 


\section{Stochastic approximation}

The utility of the Kernel Polynomial Method is that the Chebyshev moments $\mu_{m}$ may be directly estimated. The Chebyshev polynomials satisfy the recurrence relation,

$$
T_{m}(H)= \begin{cases}1 & m=0 \\ H & m=1 \\ 2 H T_{m-1}(H)-T_{m-2}(H) & m \geq 2\end{cases}
$$

Rather than calculate $\mu_{m}=\operatorname{tr} T_{m}(H)$ directly, we apply the probing approximation of Eqs. (8) and (10),

$$
\mu_{m} \approx \tilde{\mu}_{m}=\operatorname{tr} R^{\dagger} T_{m}(H) R=\operatorname{tr} R^{\dagger} \alpha_{m},
$$

where $R$ is an $N \times S$ random matrix, e.g., as defined in Eq. (11) or 15). The $N \times S$ matrices $\alpha_{m} \equiv T_{m}(H) R$ may be calculated recursively,

$$
\alpha_{m}= \begin{cases}R & m=0 \\ H R & m=1 \\ 2 H \alpha_{m-1}-\alpha_{m-2} & m \geq 2\end{cases}
$$

If we assume sparse $H$ with $\mathcal{O}(N)$ nonzero elements, each matrix-matrix multiplication requires $\mathcal{O}(N S)$ operations, and the cost to estimate all moments scales like $\mathcal{O}(N M S)$.

Substituting the approximate moments $\tilde{\mu}_{m}$ into Eq. A2 yields an approximate density of states. The corresponding free energy and electron number approximations follow from Eq. A7,

$$
\begin{gathered}
\Omega \approx \tilde{\Omega}=\sum_{m=0}^{M-1} c_{m}^{(g)} \tilde{\mu}_{m} \\
N_{e} \approx \tilde{N}_{e}=\sum_{m=0}^{M-1} c_{m}^{(f)} \tilde{\mu}_{m}
\end{gathered}
$$

with coefficients $c_{m}^{(\phi)}$ defined by Eq. A8.

\section{Appendix B: Automatic differentiation of stochastic trace estimates}

Equations A12 - A14 are the basis for a linearscaling numerical procedure to calculate the approximate free energy $\tilde{\Omega}$. The computational graph representing this recursive procedure is illustrated in Fig. 7

Using the technique of reverse-mode automatic differentiation, $12\left[35\right.$ we calculate the derivative $\mathrm{d} \tilde{\Omega} / \mathrm{d} H^{T}$ for many matrix elements simultaneously by traversing the graph backward. First, a remark on notation: We use partial derivatives $\partial z_{j}\left(z_{i}, \ldots\right) / \partial z_{i}$ in reference to the direct functional dependence of $z_{j}$ on $z_{i}$. That is, each partial derivative corresponds to a single arrow in the

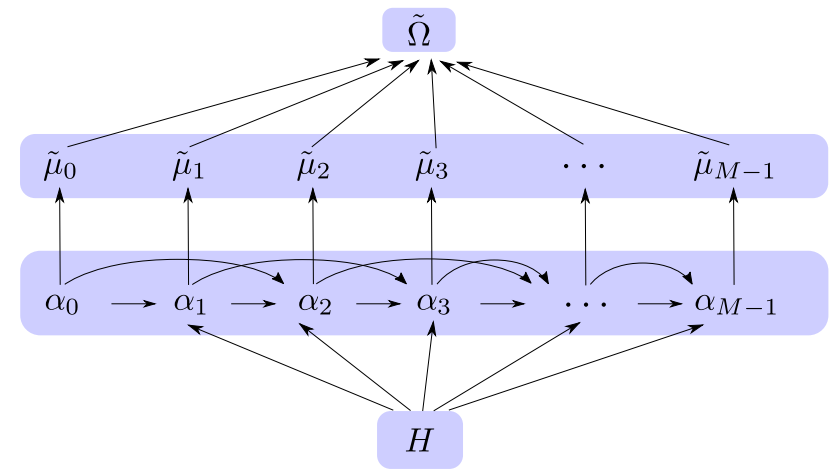

Figure 7. A graphical representation of the linear-scaling procedure to approximate the free energy $\tilde{\Omega}(H)$. This directed computational graph makes explicit all functional dependencies between intermediate terms, $H \rightarrow \alpha_{m} \rightarrow \tilde{\mu}_{m} \rightarrow \tilde{\Omega}$. Reverse-mode automatic differentiation calculates the derivative $\mathrm{d} \tilde{\Omega} / \mathrm{d} H^{T}$ for all relevant matrix elements by traversing this graph backwards.

computational graph. In contrast, we use total derivatives $\mathrm{d} \tilde{\Omega} / \mathrm{d} z_{i}$ to denote the complete transitive dependence of $\tilde{\Omega}$ on $z_{i}$. The chain rule states that $\mathrm{d} \tilde{\Omega} / \mathrm{d} H=$ $p_{1}+p_{2}+\ldots$ is a sum over all paths from $H$ to $\tilde{\Omega}$ in the graph. Each path is a chained product of partial derivatives, e.g., $p=\left(\partial \tilde{\Omega} / \partial z_{n}\right)\left(\partial z_{n} / \partial z_{n-1}\right) \ldots\left(\partial z_{1} / \partial H\right)$ where $H \rightarrow z_{1} \rightarrow z_{2} \rightarrow \ldots \tilde{\Omega}$ are connected by arrows in Fig. 7 .

Reverse-mode automatic differentiation works by expressing the desired sum-over-paths $\mathrm{d} \tilde{\Omega} / \mathrm{d} H$ using terms $\mathrm{d} \tilde{\Omega} / \mathrm{d} z_{j}$ associated with intermediate paths, $z_{j} \rightarrow z_{j+1} \rightarrow$ $\ldots \tilde{\Omega}$. We recursively move the starting point $z_{j}$ backwards along the computational graph, constructing ever longer sums-over-paths, until we finally reach the input matrix $H$. Crucially, reverse-mode automatic differentiation visits each intermediate variable $z_{j}$ only once, yet is able to produce all desired matrix elements of $\mathrm{d} \tilde{\Omega} / \mathrm{d} H$.

To explain the recursive procedure, we first consider a simple example. Suppose we have already calculated $\mathrm{d} \tilde{\Omega} / \mathrm{d} z_{3}$ and $\mathrm{d} \tilde{\Omega} / \mathrm{d} z_{2}$. Suppose further that $z_{1}$ is an intermediate variable that appears only in the functional dependencies for $z_{3}\left(z_{1}, \ldots\right)$ and $z_{2}\left(z_{1}, \ldots\right)$. Then the chain rule states $\mathrm{d} \tilde{\Omega} / \mathrm{d} z_{1}=\left(\mathrm{d} \tilde{\Omega} / \mathrm{d} z_{3}\right)\left(\partial z_{3} / \partial z_{1}\right)+$ $\left(\mathrm{d} \tilde{\Omega} / \mathrm{d} z_{2}\right)\left(\partial z_{2} / \partial z_{1}\right)$. In other words, knowing $\mathrm{d} \tilde{\Omega} / \mathrm{d} z_{3}$ and $\mathrm{d} \tilde{\Omega} / \mathrm{d} z_{2}$ allows us to calculate $\mathrm{d} \tilde{\Omega} / \mathrm{d} z_{1}$. Note that the partial derivatives $\partial z_{3} / \partial z_{1}$ and $\partial z_{2} / \partial z_{1}$ are never required by the procedure again. We keep working backwards in this way until we eventually reach $\mathrm{d} \tilde{\Omega} / \mathrm{d} H$.

Now we derive the full recursive automatic differentiation procedure. Partial derivatives (i.e., the arrows in Fig. 7f follow immediately from Eq. (A14,

$$
\frac{\partial \tilde{\Omega}}{\partial \tilde{\mu}_{m}}=c_{m}^{(g)}
$$


from Eq. A12,

$$
\frac{\partial \tilde{\mu}_{m}}{\partial \alpha_{m ; l k}}=R_{l k}^{*}
$$

and from Eq. A13,

$$
\begin{aligned}
\frac{\partial \alpha_{m+1 ; l k}}{\partial \alpha_{m ; i j}} & =2 H_{l i} \delta_{k j}, \\
\frac{\partial \alpha_{m+2 ; l k}}{\partial \alpha_{m ; i j}} & =-\delta_{l i} \delta_{k j}, \\
\frac{\partial \alpha_{m ; l k}}{\partial H_{i j}} & = \begin{cases}\delta_{l i} \alpha_{0 ; j k} & m=1 \\
2 \delta_{l i} \alpha_{m-1 ; j k} & m>1\end{cases}
\end{aligned}
$$

Our procedure to calculate $\mathrm{d} \tilde{\Omega} / \mathrm{d} H^{T}$ will require first constructing $\mathrm{d} \tilde{\Omega} / \mathrm{d} \tilde{\mu}_{m}$ and then $\mathrm{d} \tilde{\Omega} / \mathrm{d} \alpha_{m}$. Beginning at the top of Fig. 7 , there is only one path from each $\tilde{\mu}_{m}$ to $\tilde{\Omega}$, so

$$
\frac{\mathrm{d} \tilde{\Omega}}{\mathrm{d} \tilde{\mu}_{m}}=c_{m}^{(g)} .
$$

Next, we seek $\mathrm{d} \tilde{\Omega} / \mathrm{d} \alpha_{m}$, which can be factorized using recursively calculated quantities,

$$
\begin{aligned}
\frac{\mathrm{d} \tilde{\Omega}}{\mathrm{d} \alpha_{m}}= & \frac{\mathrm{d} \tilde{\Omega}}{\mathrm{d} \tilde{\mu}_{m}} \frac{\partial \tilde{\mu}_{m}}{\partial \alpha_{m}}+\sum_{l k} \frac{\mathrm{d} \tilde{\Omega}}{\mathrm{d} \alpha_{m+1 ; l k}} \frac{\partial \alpha_{m+1 ; l k}}{\partial \alpha_{m}} \\
& +\sum_{l k} \frac{\mathrm{d} \tilde{\Omega}}{\mathrm{d} \alpha_{m+2 ; l k}} \frac{\partial \alpha_{m+2 ; l k}}{\partial \alpha_{m}} .
\end{aligned}
$$

In other words, we decompose the sum-over-paths from $\alpha_{m}$ to $\tilde{\Omega}$ in terms of shorter paths which start further along the computational graph. Specifically, the shorter paths start at $\alpha_{m+1}, \alpha_{m+2}$, and $\tilde{\mu}$, because these are the three variables to which $\alpha_{m}$ contributes directly (cf. Fig. 7). To simplify the notation, we define the matrix,

$$
\beta_{m}^{*} \equiv \mathrm{d} \tilde{\Omega} / \mathrm{d} \alpha_{m} .
$$

Note that $\alpha_{m}$ does not contribute to $\tilde{\Omega}$ for $m \geq M$, so

$$
\beta_{M}=\beta_{M+1}=0 .
$$

Substitution of known quantities into Eq. (B7) yields the matrix recursion relation,

$$
\beta_{m}=c_{m}^{(g)} R+2 H \beta_{m+1}-\beta_{m+2},
$$

for $m=M-1$ down to 1 .

Finally, we obtain the desired total derivatives,

$$
\begin{aligned}
\frac{\mathrm{d} \tilde{\Omega}}{\mathrm{d} H^{T}} & =\sum_{m=1}^{M-1} \sum_{l k} \frac{\mathrm{d} \tilde{\Omega}}{\mathrm{d} \alpha_{m ; l k}} \frac{\partial \alpha_{m ; l k}}{\partial H^{T}} \\
& =\alpha_{0} \beta_{1}^{\dagger}+2 \sum_{m=1}^{M-2} \alpha_{m} \beta_{m+1}^{\dagger} .
\end{aligned}
$$

Equations B9 - B11 are the basis for a recursive procedure to calculate $\mathrm{d} \Omega / \mathrm{d} H^{T}$ for all $\mathcal{O}(N)$ elements $H_{i j}$ that contribute to $\tilde{\Omega}$. The remarkable feature of reversemode automatic differentiation is that the computational cost to calculate this full gradient scales like the cost to calculate $\tilde{\Omega}$ itself, $\mathcal{O}(N M S)$. Note that taking finite differences with respect to each element $H_{j i}$ individually would be $\mathcal{O}(N)$ times slower than automatic differentiation. Moreover, unlike finite differencing, automatic differentiation is exact up to numerical accuracy.

The traditional disadvantage of reverse-mode automatic differentiation is that it requires storage of intermediate values in the computational graph. This is apparent in Eq. (B11), which makes reference to matrices $\alpha_{m}$. Storing $\alpha_{m}$ for all $m=0, \ldots M-1$ would be a prohibitive space constraint. Our solution is to store only $\alpha_{M-2}$ and $\alpha_{M-1}$ from the forward calculation of $\tilde{\Omega}$, and then to recalculate $\alpha_{m}$ backwards, on demand, by reordering terms in Eq. A13,

$$
\alpha_{m}=2 H \alpha_{m+1}-\alpha_{m+2} .
$$

\section{Appendix C: Faster Chebyshev approximation}

Equations $\mathrm{A} 12$ and $\mathrm{A} 13$ constitute a recursive procedure to estimate the Chebyshev moments $\mu_{m} \approx \tilde{\mu}_{m}$. We can save a factor of two in the computational cost ${ }^{34}$ via the Chebyshev polynomial identity,

$$
2 T_{m}(x) T_{m^{\prime}}(x)=T_{m+m^{\prime}}(x)+T_{m-m^{\prime}}(x) .
$$

The moments $\mu_{m}=\operatorname{tr} T_{m}(H)$ may be expressed as

$$
\begin{aligned}
\mu_{2 m} & =2 \operatorname{tr} T_{m}(H) T_{m}(H)-\mu_{0}, \\
\mu_{2 m+1} & =2 \operatorname{tr} T_{m}(H) T_{m+1}(H)-\mu_{1},
\end{aligned}
$$

Because $H$ is Hermitian, approximate moments $\tilde{\mu}_{m}=$ $\operatorname{tr} R^{\dagger} T_{m}(H) R$ may be expressed as

$$
\begin{aligned}
\tilde{\mu}_{2 m} & =2 \operatorname{tr} \alpha_{m}^{\dagger} \alpha_{m}-\tilde{\mu}_{0}, \\
\tilde{\mu}_{2 m+1} & =2 \operatorname{tr} \alpha_{m}^{\dagger} \alpha_{m+1}-\tilde{\mu}_{1},
\end{aligned}
$$

where $\alpha_{m} \equiv T_{m}(H) R$ are computed from Eq. A13). We compute the first two moments directly, $\tilde{\mu}_{0}=\operatorname{tr} R^{\top} R$ and $\tilde{\mu}_{1}=\operatorname{tr} R^{\dagger} H R$, without involving $\alpha_{0}$ or $\alpha_{1}$.

As before, the free energy approximation $\tilde{\Omega}$ is given by Eq. A14. We require half the number of $\alpha_{m}$ matrices by using Eqs. (C4) and (C5) instead of Eq. A12.

In analogy with Appendix $\mathrm{B}$, we transform this recursive procedure to calculate $\tilde{\Omega}$ into one that calculates its gradient. After a lengthy derivation ${ }^{66}$ we obtain a recursion relation for matrices $\beta_{m}^{*} \equiv \mathrm{d} \tilde{\Omega} / \mathrm{d} \alpha_{m}$ starting from

$$
\beta_{M / 2+1}=0 \quad \text { and } \quad \beta_{M / 2}=2 c_{M-1}^{(g)} \alpha_{M / 2-1},
$$

and working down to $m=1$ via, 


$$
\begin{gathered}
\beta_{m}=[m>1] 2 c_{2 m-1}^{(g)} \alpha_{m-1}+4 c_{2 m}^{(g)} \alpha_{m}+2 c_{2 m+1}^{(g)} \alpha_{m+1} \\
+2 H \beta_{m+1}-\beta_{m+2} .
\end{gathered}
$$

Above we use the Iverson bracket notation,

$$
[P]=\left\{\begin{array}{ll}
1 & \text { if } P \\
0 & \text { otherwise }
\end{array} .\right.
$$

Our result for the gradient of approximate free energy is

$$
\begin{array}{r}
\frac{\mathrm{d} \tilde{\Omega}}{\mathrm{d} H^{T}}=c_{1}^{(g)} R R^{\dagger}-\sum_{m=1}^{M / 2-1} c_{2 m+1}^{(g)} R R^{\dagger} \\
+\alpha_{0} \beta_{1}^{\dagger}+2 \sum_{m=1}^{M / 2-1} \alpha_{m} \beta_{m+1}^{\dagger} .
\end{array}
$$

The forward procedure involving Eqs. (C4) and C5 is correct only if $H$ is Hermitian. When differentiating this procedure, perturbations to the Hamiltonian $H \mapsto H+\mathrm{d} H$ should also be Hermitian. We must explicitly symmetrize Eq. C9, the output of automatic differentiation, to get the correct final result.

\section{Appendix D: Ensemble of fixed electron number}

So far we have been working with the grand potential $\Omega$ at fixed chemical potential $\mu$. For numerical calculations of finite systems, it is often preferable to work in the canonical ensemble where $\mu$ varies according to a fixed electron number $N_{e}$. To calculate $\mu\left(N_{e}\right)$, we use the bisection method to invert the probing estimate $N_{e}(\mu) \approx \sum_{m} c_{m}^{(f)} \tilde{\mu}_{m}$ of Eq. A15. The coefficients $c_{m}^{(f)}$ involve an integral over the Fermi function and must be recalculated for each trial value of the chemical potential $\mu$. The dominant computational cost, however, is in approximating the Chebyshev moments $\tilde{\mu}_{m} \approx \mu_{m}$. Fortunately, the moments are independent of chemical potential $\mu$ and thus do not need to be recalculated during the search. The bisection method is guaranteed to converge because $\rho(x) \approx \rho_{M}(x)$ is a strictly positive approximation when using the damping coefficients of Eq. A6.

The relevant free energy in the canonical ensemble, $F\left(N_{e}\right)$, is related to the grand potential by a Legendre transform,

$$
F\left(N_{e}\right)=\Omega(\mu)+\mu N_{e}
$$

The general thermodynamic relation,

$$
\frac{\mathrm{d} \Omega}{\mathrm{d} \mu}=-N_{e}
$$

can be verified in our context using Eqs. (2), (3), and (6). The differential of $\Omega(\mu)$ at fixed $N_{e}$ then becomes

$$
\left.\mathrm{d} \Omega\right|_{N_{e}}=\left.\frac{\mathrm{d} \Omega}{\mathrm{d} \mu} \mathrm{d} \mu\right|_{N_{e}}+\left.\mathrm{d} \Omega\right|_{\mu}=-\left.N_{e} \mathrm{~d} \mu\right|_{N_{e}}+\left.\mathrm{d} \Omega\right|_{\mu} .
$$

We take the differential of both sides of Eq. (D1), at fixed $N_{e}$, and substitute Eq. (D3) to obtain

$$
\left.\mathrm{d} F\right|_{N_{e}}=\left.\mathrm{d} \Omega\right|_{\mu}
$$

Thus, in both ensembles, the derivative of the relevant free energy with respect to the Hamiltonian yields the density matrix $f(H)$,

$$
\left.\frac{\mathrm{d} F}{\mathrm{~d} H^{T}}\right|_{N_{e}}=\left.\frac{\mathrm{d} \Omega}{\mathrm{d} H^{T}}\right|_{\mu}=f(H) .
$$

In a numerical calculation, for which we approximate $\Omega \approx$ $\operatorname{tr} g(H) R R^{\dagger}$, we should also substitute $N_{e} \approx \operatorname{tr} f(H) R R^{\dagger}$ in the definition of $F$ in Eq. (D1). This way the thermodynamic identity of Eq. (D4) continues to hold exactly, with or without the probing approximation.

\section{Appendix E: Error analysis for gradient-based probing}

\section{Main result}

In Sec. III we argued that gradient-based probing

$$
f(H)=\frac{\mathrm{d}}{\mathrm{d} H^{T}} \operatorname{tr} g(H) R R^{\dagger}+\Delta f^{\operatorname{grad}}(H)
$$

yields better density matrix estimates than does direct probing. Here we derive the asymptotic scaling of the gradient-based probing error, $\Delta f(H)^{\mathrm{grad}}$, valid for zerotemperature metals. Using the fact that $\left(R R^{\dagger}\right)_{i i}=1$, we can rewrite Eq. 30 in an explicit form,

$$
\Delta f(H)_{i j}^{\mathrm{grad}}=\sum_{m \neq n} \frac{\partial g(H)_{m n}}{\partial H_{j i}}\left(R R^{\dagger}\right)_{n m} .
$$

If the matrix elements of $R$ are uncorrelated and defined according to Eq. 11, the variance of the error is

$$
\operatorname{var}\left[\Delta f(H)_{i j}^{\operatorname{grad}}\right]=\frac{1}{S} \sum_{m<n}\left|\frac{\partial g(H)_{m n}}{\partial H_{j i}}\right|^{2}
$$

in analogy to Eq. (12). Note that the sum cannot contribute any $S$ dependence, so $\Delta f(H)_{i j}^{\text {grad }} \sim S^{-1 / 2}$ in the uncorrelated case.

If $R$ is optimized according to Eq. [15, we instead have

$$
\operatorname{var}\left[\Delta f(H)_{i j}^{\mathrm{grad}}\right]=\sum_{m<n} \delta_{c(m), c(n)}\left|\frac{\partial g(H)_{m n}}{\partial H_{j i}}\right|^{2},
$$

in analogy to Eq. (17). The constraint $c(m)=c(n)$ is only satisfied for orbitals $m$ and $n$ whose real-space distance satisfies $r_{m n} \gtrsim S^{1 / d}$, and thus introduces a nontrivial dependence on $S$.

For insulators and for systems at finite temperature, $g(H)_{m n}$ decays exponentially in $r_{m n}=\left|\mathbf{r}_{m}-\mathbf{r}_{n}\right|$. As a consequence, $\Delta f(H)_{i j}^{\text {grad }}$ decays exponentially in $S^{1 / d}$ for 
spatial dimension $d$. Metals at zero temperature, however, give rise to universal power law decay. We focus our analysis on these systems because they represent a worst-case scenario. We saw in Eq. 22 that $g(H)_{m n}$ decays like $r_{m n}^{-(d+3) / 2}$. As we will show in Appendix E 2. the corresponding matrix derivative also decays polynomially,

$$
\frac{\partial g(H)_{m n}}{\partial H_{j i}} \sim \frac{1}{\left(r_{m j} r_{n i}\right)^{\frac{d-1}{2}}\left(r_{m j}+r_{n i}\right)^{2}} .
$$

This decay is valid when $r_{m j}=\left|\mathbf{r}_{m}-\mathbf{r}_{j}\right|$ and $r_{n i}=\mid \mathbf{r}_{n}$ $\mathbf{r}_{i} \mid$ are large compared to the inverse Fermi momentum, $k_{F}^{-1}$.

When $S$ is large, we can work in the continuum limit to calculate

$$
\begin{aligned}
& \operatorname{var}\left[\Delta f(H)_{i j}^{\operatorname{grad}}\right] \sim \\
& \quad \frac{1}{S} \int \mathrm{d}^{d} \mathbf{r}_{m} \mathrm{~d}^{d} \mathbf{r}_{n} \Theta\left(r_{m n}-S^{\frac{1}{d}}\right)\left|\frac{\partial g(H)_{m n}}{\partial H_{j i}}\right|^{2} .
\end{aligned}
$$

The Heaviside step function $\Theta\left(r_{m n}-S^{1 / d}\right)$ encodes the fact that orbitals $m$ and $n$ can only contribute if separated by distance $r_{m n} \gtrsim S^{1 / d}$. The outer factor of $S^{-1}$ encodes the fact that arbitrary indices $m$ and $n$ only have the same color with probability $S^{-1}$. This uniform probabilistic treatment of the integrand is justified because $\partial g(H)_{m n} / \partial H_{j i}$ decays sufficiently slowly.

Our interest is estimation of local elements $f(H)_{i j}$, for which $r_{i j}=\left|\mathbf{r}_{i}-\mathbf{r}_{j}\right| \ll S^{1 / d}$ in the large $S$ limit. Without loss of generality, we may take $i=j=0$ in our scaling calculation. Substitution of Eq. E5 yields

$$
\begin{aligned}
\operatorname{var}\left[\Delta f(H)_{i j}^{\mathrm{grad}}\right] & \sim \\
& \frac{1}{S} \iint \mathrm{d}^{d} \mathbf{r}_{m} \mathrm{~d}^{d} \mathbf{r}_{n} \frac{\Theta\left(r_{m n}-S^{1 / d}\right)}{\left(r_{m} r_{n}\right)^{d-1}\left(r_{m}+r_{n}\right)^{4}} .
\end{aligned}
$$

Because $S^{1 / d}$ is the only length scale in the integral, we can perform dimensional analysis to find our final result,

$$
\operatorname{var}\left[\Delta f(H)_{j i}^{\operatorname{grad}}\right] \sim S^{-(d+2) / d} .
$$

The behavior of $\partial g(H)_{m n} / \partial H_{00}$ as $r_{m} \rightarrow 0$ and $r_{n} \rightarrow 0$ is not pertinent to this scaling result. Indeed, if we modify Eq. (E7) to constrain $r_{m}>\sigma$ and $r_{n}>\sigma$ for some length scale $\sigma$ that satisfies $k_{F}^{-1} \ll \sigma \ll S^{1 / d}$, then Eq. (E8) still holds.

\section{Asymptotic decay of the energy matrix derivative}

Here we derive the asymptotic decay of $\partial g(H)_{m n} / \partial H_{i j}$ for a model metallic Hamiltonian. tion,

We begin with the representation of the Dirac- $\delta$ func-

$$
\delta\left(x-\epsilon_{\mathbf{k}}\right)=-\frac{1}{\pi} \operatorname{Im} \frac{1}{x-\epsilon_{\mathbf{k}}+i \eta} \quad \eta \rightarrow 0^{+} .
$$

This identity generalizes to a matrix equation for Hermitian $H$,

$$
\delta(x-H)=\frac{i}{2 \pi}\left[G^{+}(x)-G^{-}(x)\right] .
$$

The retarded/advanced Green's functions are defined as

$$
G^{ \pm}(x)=\frac{1}{x-H \pm i \eta} .
$$

We employ finite $\eta$ to regularize intermediate calculations, with the understanding that eventually $\eta \rightarrow 0^{+}$.

Equation (E10) yields a differentiable representation of the energy matrix,

$$
g(H)=\frac{i}{2 \pi} \int_{-\infty}^{+\infty} \mathrm{d} x g(x)\left[G^{+}(x)-G^{-}(x)\right] .
$$

We seek the derivative with respect to an arbitrary matrix element $H_{i j}$. For any invertible operator $B$ we have $B^{-1} \partial_{\alpha}\left(B B^{-1}\right)=0$; applying the product rule, we conclude that $\partial_{\alpha} B^{-1}=-B^{-1}\left(\partial_{\alpha} B\right) B^{-1}$. Substituting $B^{-1} \mapsto G^{ \pm}$and $\alpha \mapsto H_{i j}$, we find

$$
\frac{\partial G^{ \pm}(x)}{\partial H_{i j}}=G^{ \pm}(x) \Delta^{i j} G^{ \pm}(x),
$$

where $\Delta^{i j}=\partial H / \partial H_{i j}$ is the matrix with real-space elements $\Delta_{m n}^{i j}=\delta_{i m} \delta_{j n}$. Equivalence to the Dyson equation, 67 at first order in the perturbation $\epsilon \Delta$, is apparent after expanding $\partial G^{ \pm} / \partial H_{i j} \approx\left(G_{H+\epsilon \Delta}^{ \pm}-G_{H}^{ \pm}\right) / \epsilon$.

The above identities are valid for any Hamiltonian. Now we focus on a translation invariant Hamiltonian $H=H_{0}$ with quadratic dispersion $\epsilon_{\mathbf{k}}=k^{2} / 2$ that is filled to chemical potential $\mu=k_{F}^{2} / 2$. In momentumspace, the non-interacting Green's functions are

$$
G_{0}^{ \pm}(x)=\int \mathrm{d}^{d} \mathbf{k} \frac{1}{x-\epsilon_{\mathbf{k}} \pm i \eta}|\mathbf{k}\rangle\langle\mathbf{k}| .
$$

The $\mathbf{k}$ integrals run over the first Brillouin zone. The eigenstates $|\mathbf{k}\rangle$ have real-space representation

$$
\left\langle\mathbf{r}_{i} \mid \mathbf{k}\right\rangle=\frac{1}{(2 \pi)^{d / 2}} e^{i \mathbf{k} \cdot \mathbf{r}_{i}} .
$$

We have assumed that the volume of the primitive cell is one.

We evaluate Eq. E13 at $H=H_{0}$. Inserting $\Delta^{i j}=$ $\left|\mathbf{r}_{i}\right\rangle\left\langle\mathbf{r}_{j}\right|$, we find matrix elements

$$
\begin{aligned}
& \frac{\partial G^{ \pm}(x)_{m n}}{\partial H_{i j}}=\left\langle\mathbf{r}_{m}\left|G_{0}^{ \pm}(x)\right| \mathbf{r}_{i}\right\rangle\left\langle\mathbf{r}_{j}\left|G_{0}^{ \pm}(x)\right| \mathbf{r}_{n}\right\rangle \\
& \quad=\frac{1}{(2 \pi)^{2 d}} \iint \mathrm{d}^{d} \mathbf{k} \mathrm{d}^{d} \mathbf{k}^{\prime} \frac{e^{i \mathbf{k} \cdot \mathbf{r}_{m i}-i \mathbf{k}^{\prime} \cdot \mathbf{r}_{n j}}}{\left(x-\epsilon_{\mathbf{k}} \pm i \eta\right)\left(x-\epsilon_{\mathbf{k}^{\prime}} \pm i \eta\right)} .
\end{aligned}
$$

where $\mathbf{r}_{m i}=\mathbf{r}_{m}-\mathbf{r}_{i}$ and $\mathbf{r}_{n j}=\mathbf{r}_{n}-\mathbf{r}_{j}$. 
Taking the derivative of both sides of Eq. E12, we find

$$
\begin{aligned}
\frac{\partial g(H)_{m n}}{\partial H_{i j}}=\frac{1}{(2 \pi)^{2 d}} & \int \mathrm{d} x g(x) \iint \mathrm{d}^{d} \mathbf{k} \mathrm{d}^{d} \mathbf{k}^{\prime} \\
& \times e^{i \mathbf{k} \cdot \mathbf{r}_{m i}-i \mathbf{k}^{\prime} \cdot \mathbf{r}_{n j}} W_{\mathbf{k k}^{\prime}}(x)
\end{aligned}
$$

where, in the eventual limit that $\eta \rightarrow 0^{+}$,

$$
\begin{aligned}
W_{\mathbf{k k}^{\prime}}(x) & =-\frac{1}{\pi} \operatorname{Im} \frac{1}{\left(x-\epsilon_{\mathbf{k}}+i \eta\right)\left(x-\epsilon_{\mathbf{k}^{\prime}}+i \eta\right)} \\
& =\operatorname{Re}\left[\frac{\delta\left(x-\epsilon_{\mathbf{k}}\right)}{x-\epsilon_{\mathbf{k}^{\prime}}+i \eta}+\frac{\delta\left(x-\epsilon_{\mathbf{k}^{\prime}}\right)}{x-\epsilon_{\mathbf{k}}+i \eta}\right] .
\end{aligned}
$$

The last equality follows from the identity

$$
\operatorname{Im}(a b)=\operatorname{Im}(a) \operatorname{Re}(b)+\operatorname{Im}(b) \operatorname{Re}(a),
$$

and application of Eq. (E9), which is valid up to irrelevant corrections for small $\eta$.

Substitution yields

$$
\begin{aligned}
\frac{\partial g(H)_{m n}}{\partial H_{i j}} & =\frac{1}{(2 \pi)^{2 d}} \iint \mathrm{d}^{d} \mathbf{k} \mathrm{d}^{d} \mathbf{k}^{\prime} e^{i \mathbf{k} \cdot \mathbf{r}_{m i}-i \mathbf{k}^{\prime} \cdot \mathbf{r}_{n j}} \operatorname{Re}\left[\frac{g\left(\epsilon_{\mathbf{k}}\right)}{\epsilon_{\mathbf{k}}-\epsilon_{\mathbf{k}^{\prime}}+i \eta}+\frac{g\left(\epsilon_{\mathbf{k}^{\prime}}\right)}{\epsilon_{\mathbf{k}^{\prime}}-\epsilon_{\mathbf{k}}+i \eta}\right] \\
& =\frac{2}{(2 \pi)^{d}} \int \mathrm{d}^{d} \mathbf{k} g\left(\epsilon_{\mathbf{k}}\right)\left[e^{i \mathbf{k} \cdot \mathbf{r}_{m i}} \operatorname{Re} \mathcal{G}_{0}^{+}\left(k, r_{n j}\right)+e^{-i \mathbf{k} \cdot \mathbf{r}_{n j}} \operatorname{Re} \mathcal{G}_{0}^{+}\left(k, r_{m i}\right)\right]
\end{aligned}
$$

The non-interacting Green's function integrals are,

$$
\mathcal{G}_{0}^{+}(k, r)=\frac{1}{(2 \pi)^{d}} \int_{\mathbb{R}^{d}} \mathrm{~d}^{d} \mathbf{k}^{\prime} \frac{e^{i \mathbf{k}^{\prime} \cdot \mathbf{r}}}{k^{2}-k^{\prime 2}+i \eta}= \begin{cases}-\frac{i}{2 \sqrt{k^{2}+i \eta}} e^{i \sqrt{k^{2}+i \eta} r} & d=1 \\ -\frac{i}{4} H_{0}^{(1)}\left(\sqrt{k^{2}+i \eta} r\right) & d=2 \\ -\frac{1}{4 \pi r} e^{i \sqrt{k^{2}+i \eta} r} & d=3\end{cases}
$$

where $H_{0}^{(1)}$ is the Hankel function of the first kind. Above we have extended the integration domain from the first Brillouin zone to $\mathbb{R}^{d}$; this continuum limit (lattice parameter $a \rightarrow 0$ ) is valid when $a k_{F} \ll 1$.

We seek to evaluate Eq. E20 in the limit $\eta \rightarrow 0^{+}$. Note that $\mathcal{G}_{0}^{+}(k=0, r)$ is singular in this limit for $d=1$ and $d=2$. In one dimension, we use the fact that $\operatorname{Im}\left(k^{2}+i \eta\right)^{-1 / 2}=-\pi \delta(k) / 2$. In two dimensions, the $\left(k^{2}+i \eta\right)^{-1 / 4}$ singularity at $\mathcal{G}_{0}^{+}(k=0, r)$ will be scaled by a factor of $k J_{0}(k r) \sim \sqrt{k}$ in the integrand, and can be ignored. Thus, when $\eta \rightarrow 0^{+}$, we employ

$$
\operatorname{Re} \mathcal{G}_{0}^{+}(k, r)= \begin{cases}-\frac{\pi}{4} \delta(k)+\frac{\sin (k r)}{2 k} & d=1 \\ \frac{1}{4} Y_{0}(k r) & d=2 \\ -\frac{\cos (k r)}{4 \pi r} & d=3\end{cases}
$$

where $Y_{0}(k r)$ is the Bessel function of the second kind. Substitution into Eq. E20 with $g\left(\epsilon_{\mathbf{k}}\right)=\left(k^{2}-k_{F}^{2}\right) \Theta\left(k_{F}-k\right) / 2$ yields

$$
\begin{aligned}
& \left.\frac{\partial g(H)_{m n}}{\partial H_{i j}}\right|_{d=1}=\frac{k_{F}^{2}}{4}+\frac{1}{2 \pi} \int_{0}^{k_{F}} \mathrm{~d} k\left(k^{2}-k_{F}^{2}\right) \frac{\sin \left[k\left(r_{m i}+r_{n j}\right)\right]}{k} \approx \frac{\sin \left[k_{F}\left(r_{m i}+r_{n j}\right)\right]}{\pi\left(r_{m i}+r_{n j}\right)^{2}}, \\
& \left.\frac{\partial g(H)_{m n}}{\partial H_{i j}}\right|_{d=2}=\frac{1}{4} \int_{0}^{k_{F}} \mathrm{~d} k k\left(k^{2}-k_{F}^{2}\right)\left[J_{0}\left(k r_{m i}\right) Y_{0}\left(k r_{n j}\right)+J_{0}\left(k r_{n j}\right) Y_{0}\left(k r_{m i}\right)\right] \approx k_{F} \frac{\cos \left[k_{F}\left(r_{m i}+r_{n j}\right)\right]}{\sqrt{r_{m i} r_{n j}}\left(r_{m i}+r_{n j}\right)^{2}} \\
& \left.\frac{\partial g(H)_{m n}}{\partial H_{i j}}\right|_{d=3}=-\frac{1}{8 \pi^{3} r_{m i} r_{n j}} \int_{0}^{k_{F}} \mathrm{~d} k k\left(k^{2}-k_{F}^{2}\right) \sin \left[k\left(r_{m i}+r_{n j}\right)\right] \approx-k_{F}^{2} \frac{\sin \left[k_{F}\left(r_{m i}+r_{n j}\right)\right]}{4 \pi^{3} r_{m i} r_{n j}\left(r_{m i}+r_{n j}\right)^{2}} .
\end{aligned}
$$

The final three approximations are asymptotically valid when $r_{m i}$ and $r_{n j}$ are large compared to $k_{F}^{-1}$. We conclude, in all dimensions $d$, that the matrix derivative de- cays as

$$
\frac{\partial g(H)_{m n}}{\partial H_{i j}} \sim \frac{1}{\left(r_{m i} r_{n j}\right)^{\frac{d-1}{2}}\left(r_{m i}+r_{n j}\right)^{2}}
$$


for large $r_{m i}$ and $r_{n j}$. This power law decay is universal and we have verified it numerically in the context of simple tight-binding models.

\section{REFERENCES}

${ }^{1}$ M. Elstner, D. Porezag, G. Jungnickel, J. Elsner, M. Haugk, T. Frauenheim, S. Suhai, and G. Seifert, Phys. Rev. B 58, 7260 (1998)

${ }^{2}$ M. Elstner and G. Seifert, Phil. Trans. R. Soc. A 372 (2014)

${ }^{3}$ B. Aradi, A. M. N. Niklasson, and T. Frauenheim, J. Chem. Theory Comput. 11, 3357 (2015).

${ }^{4}$ S. Doniach, Physica B+C 91, 231 (1977).

${ }^{5}$ L. M. Falicov and J. C. Kimball, Phys. Rev. Lett. 22, 997 (1969).

${ }^{6}$ P. G. de Gennes, Superconductivity of Metals and Alloys (Benjamin, New York, 1966).

${ }^{7}$ S. Goedecker and L. Colombo, Phys. Rev. Lett. 73, 122 (1994)

${ }^{8}$ A. F. Voter, J. D. Kress, and R. N. Silver, Phys. Rev. B 53, 12733 (1996)

${ }^{y}$ J. VandeVondele, U. Borštnik, and J. Hutter, J. Chem. Theory Comput. 8, 3565 (2012)

${ }^{10}$ M. J. Cawkwell and A. M. N. Niklasson, J. Chem. Phys. 137 (2012).

${ }^{11}$ G.-W. Chern, K. Barros, C. D. Batista, J. D. Kress, and G. Kotliar, Phys. Rev. Lett. 118, 226401 (2017)

${ }^{12}$ K. Barros and Y. Kato, Phys. Rev. B 88, 235101 (2013).

${ }^{13}$ K. Barros, J. W. F. Venderbos, G.-W. Chern, and C. D. Batista, Phys. Rev. B 90, 245119 (2014).

${ }^{14}$ R. Ozawa, S. Hayami, K. Barros, G.-W. Chern, Y. Motome, and C. D. Batista, J. Phys. Soc. Jpn. 85, 103703 (2016)

${ }^{15}$ Z. Wang, K. Barros, G.-W. Chern, D. L. Maslov, and C. D. Batista, Phys. Rev. Lett. 117, 206601 (2016)

${ }^{16}$ R. Ozawa, S. Hayami, and Y. Motome, Phys. Rev. Lett. 118, 147205 (2017)

${ }^{1 /}$ R. Ozawa, S. Hayami, K. Barros, and Y. Motome, Phys. Rev. B 96, 094417 (2017)

${ }^{18}$ G.-W. Chern, K. Barros, Z. Wang, H. Suwa, and C. D. Batista, Phys. Rev. B 97, 035120 (2018)

${ }^{19}$ W. Kohn, Phys. Rev. 115, 809 (1959)

${ }^{20}$ S. Goedecker, Rev. Mod. Phys. 71, 1085 (1999)

${ }^{21}$ D. R. Bowler and T. Miyazaki, Rep. Prog. Phys. 75, 036503 (2012)

${ }^{22}$ G. Beylkin, N. Coult, and M. J. Mohlenkamp, J. Comput. Phys. 152, 32 (1999)

${ }^{23}$ A. M. N. Niklasson, Phys. Rev. B 66, 155115 (2002)

${ }^{24}$ A. M. N. Niklasson, Phys. Rev. B 68, 233104 (2003).

${ }^{25}$ A. R. Curtis, M. J. D. Powell, and J. K. Reid, J. Inst. Math. App. 13, 117 (1974)

${ }^{20}$ T. F. Coleman and J. J. Moré, SIAM J. Numer. Anal. 20, 187 (1983)

${ }^{27}$ C. Bekas, E. Kokiopoulou, and Y. Saad, Appl. Numer. Math. 57, 1214 (2007)

${ }^{2 \succ}$ J. M. Tang and Y. Saad, Numer. Linear Algebra Appl. 19, 485 (2012)

${ }^{29}$ F. R. Krajewski and M. Parrinello, Phys. Rev. B 73, 041105 (2006)

ऊv Y. Luo, A. Zen, and S. Sorella, J. Chem. Phys. 141, 194112 (2014).

${ }^{31}$ E. Arnon, E. Rabani, D. Neuhauser, and R. Baer, J. Chem. Phys. 146, 224111 (2017)
${ }^{32}$ R. N. Silver and H. Röder, Int. J. Mod. Phys. C 5, 735 (1994).

${ }^{33}$ R. N. Silver, H. Röder, A. F. Voter, and J. D. Kress, J. Comput. Phys. 124, 115 (1996).

${ }^{34}$ A. Weiße, G. Wellein, A. Alvermann, and H. Fehske, Rev. Mod. Phys. 78, 275 (2006).

${ }^{35}$ A. Griewank, in Mathematical Programming: Recent Developments and Applications, edited by M. Iri and K. Tanabe (Kluwer Academic, Dordrecht, The Netherlands, 1989) pp. 83-108.

${ }^{36}$ M. Ceriotti, T. D. Kühne, and M. Parrinello, J. Chem. Phys. 129, 024707 (2008).

${ }^{37}$ R. B. Sidje and Y. Saad, Numer. Algor. 56, 455 (2011)

${ }^{38}$ L. Lin, Y. Saad, and C. Yang, SIAM Review 58, 34 (2016)

${ }^{39}$ L. Lin, Numer. Math. 136, 183 (2017)

${ }^{40}$ T. Iitaka and T. Ebisuzaki, Phys. Rev. E 69, 057701 (2004)

${ }^{41} \mathrm{D}$. Girard, Un algorithme simple et rapide pour la validation croisée généralisée sur des problèmes de grande taille, Tech. Rep. RR 669-M (Informatique et Mathématiques Appliquées de Grenoble, Grenoble, France, 1987).

${ }^{42}$ M. Hutchinson, Comm. Stat. Simul. Comput. 19, 433 (1990)

${ }^{43}$ J. Skilling, "Maximum entropy and bayesian methods," (Kluwer, Dordrecht, 1989) p. 455.

${ }^{44}$ D. A. Drabold and O. F. Sankey, Phys. Rev. Lett. 70, 3631 (1993)

${ }^{45}$ L.-W. Wang, Phys. Rev. B 49, 10154 (1994)

${ }^{46}$ H. Röder, R. N. Silver, D. A. Drabold, and J. J. Dong, Phys. Rev. B 55, 15382 (1997)

${ }^{47}$ R. Baer, D. Neuhauser, and E. Rabani, Phys. Rev. Lett. 111, 106402 (2013).

${ }^{48}$ D. Neuhauser, Y. Gao, C. Arntsen, C. Karshenas, E. Rabani, and R. Baer, Phys. Rev. Lett. 113, 076402 (2014).

${ }^{49}$ Y. Gao, D. Neuhauser, R. Baer, and E. Rabani, J. Chem. Phys. 142, 034106 (2015)

${ }^{50}$ E. Rabani, R. Baer, and D. Neuhauser, Phys. Rev. B 91, 235302 (2015)

${ }^{5}$ Y. Cytter, E. Rabani, D. Neuhauser, and R. Baer, ArXiv e-prints (2018), arXiv:1801.02163 [cond-mat.mtrl-sci]

${ }^{52}$ S. Vembu, Q. J. Math 12, 165 (1961)

${ }^{53}$ K. Barros and W. Klein, J. Chem. Phys. 139, 174505 (2013).

${ }^{54}$ S. Goedecker, Phys. Rev. B 58, 3501 (1998).

${ }^{55}$ S. Ismail-Beigi and T. A. Arias, Phys. Rev. Lett. 82, 2127 (1999)

${ }^{56}$ L. He and D. Vanderbilt, Phys. Rev. Lett. 86, 5341 (2001)

${ }^{57}$ S. N. Taraskin, D. A. Drabold, and S. R. Elliott, Phys. Rev. Lett. 88, 196405 (2002)

${ }^{58}$ J. Jędrzejewski and T. Krokhmalskii, Phys. Rev. B 70, 153102 (2004)

${ }^{59}$ M. Benzi, P. Boito, and N. Razouk, SIAM Review 55, 3 (2013)

${ }^{60} \mathrm{In}$ actual practice, we use a symmetrized probing approximation for the density matrix: $f(H) \approx\left[f(H) R R^{\dagger}+R R^{\dagger} f(H)\right] / 2$.

${ }^{61}$ L. Lin, A. García, G. Huhs, and C. Yang, J. Phys.: Condens. Matter 26, 305503 (2014).

${ }^{02}$ C. Lanczos, J. Res. Nat. Bur. Stand. 45, 255 (1950).

${ }^{63}$ D. Jackson, Ph.D. thesis, Georg-August-Universität Göttingen (1911).

${ }^{64}$ D. Jackson, Trans. Am. Math. Soc. 13, 491 (1912).

${ }^{65}$ M. Abramowitz and I. A. Stegun, eds., Handbook of Mathematical Functions (Dover, New York, 1972).

${ }^{66}$ This automatic differentiation procedure is subtle because the relevant intermediate derivatives are formally non-analytic, and make sense only when variations to the Hamiltonian $\mathrm{d} H$ are constrained to be Hermitian.

${ }^{67}$ E. N. Economou, Green's functions in quantum physics, 3rd ed. (Springer, 2006). 